Cahiers
de a 2 Recherche
sur les Droits

Cahiers de la recherche sur les droits fondamentaux

16 | 2018

Les partis politiques

\title{
Les partis politiques en France : des organismes de droit privé ?
}

Political Parties in France, Can They Be Considered to Be Private-Law Bodies?

Jean-Pierre Camby

\section{(2) OpenEdition}

\section{Journals}

Édition électronique

URL : https://journals.openedition.org/crdf/303

DOI : $10.4000 /$ crdf.303

ISSN : 2264-1246

Éditeur

Presses universitaires de Caen

Édition imprimée

Date de publication : 16 novembre 2018

Pagination : $31-34$

ISBN : 978-2-84133-901-3

ISSN : $1634-8842$

\section{Référence électronique}

Jean-Pierre Camby, "Les partis politiques en France: des organismes de droit privé ? ", Cahiers de la recherche sur les droits fondamentaux [En ligne], 16 | 2018, mis en ligne le 16 novembre 2019, consulté le 14 novembre 2022. URL : http://journals.openedition.org/crdf/303 ; DOI : https://doi.org/10.4000/ crdf.303

Tous droits réservés 


\title{
Les partis politiques en France: des organismes de droit privé?
}

\author{
Jean-Pierre CAMBY \\ Professeur associé à l'université de Versailles Saint-Quentin-en-Yvelines
}

\author{
I. L'absence de définition constitutionnelle, \\ garantie du libre exercice de l'action des partis politiques \\ II. Les partis politiques, organismes de droit privé, \\ entretiennent des rapports de droit privé avec leurs membres
}

Les partis politiques sont entrés à grand-peine dans la Constitution de 1958. Il est vrai que le général de Gaulle ne les appréciait guère, fustigeant, dans sa conférence de presse du 18 octobre 1962, leur opposition à la réforme et leur souhait de retour en arrière: «[...] comme le voudraient tous les anciens partis afin de rétablir le régime de malheur». Le régime de "malheur» est évidemment celui des III ${ }^{\mathrm{e}}$ et IV ${ }^{\mathrm{e}}$ Républiques, où les partis sont ainsi accusés d'être les auteurs, sous-jacents mais bien réels, du jeu parlementaire, via des institutions qui ne canalisent que faiblement le risque que courent des exécutifs fragilisés, confrontés aux débats parlementaires qui favorisent les alliances politiques temporaires et la concentration des pouvoirs au profit des assemblées. Beaucoup d'éléments contribuent à la crise gouvernementale et à l'instabilité: l'absence de contrôle de constitutionnalité qui s'explique par l'assimilation de la souveraineté de la loi avec celle du Parlement, la désuétude de la dissolution sous la III ${ }^{\mathrm{e}}$ République, sa difficulté de mise en œuvre sous la $\mathrm{IV}^{\mathrm{e}}$, les modes de scrutin, les coalitions politiques. Ainsi est-il facile de dénoncer les difficultés engendrées par la « République des partis», les cursus politiques qu'expliquent un «scrutin de retraités ", comme Édouard Herriot qualifiait la représentation proportionnelle, la faible discipline de vote qui favorise des «majorités d'idées » dans lesquelles les partis se meuvent aisément.

Les partis étaient ainsi accusés régulièrement d'être responsables des maux qui sont reprochés aux institu- tions. On a beau dire que les institutions elles-mêmes, qui conduisent presque mécaniquement à une concentration des pouvoirs, jouent un grand rôle dans la fragilité des exécutifs, les «forces partisanes» sont citées à comparaittre au banc des accusés, au premier rang. La $\mathrm{V}^{\mathrm{e}}$ République avait sans doute mis fin à cette approche, puisqu'elle a entraîné une nouvelle structuration des partis politiques, principalement en fonction des élections présidentielle et législatives. Il reste que des courants d'opinion naissants, ou du moins de nouvelles attentes sociétales, peinent à s'incarner dans des partis nouveaux ou à être intégrés dans la logique de partis plus anciens et que les primaires de 2017, qui représentent une sorte sinon d'institutionnalisation, du moins d'insertion du rôle des organisations partisanes dans le processus électoral, ont été, pour ceux des candidats qui y ont eu recours, un échec. L'élection d'Emmanuel Macron démontre, à l'inverse, qu'un relais par un parti politique est indispensable pour accompagner la victoire, surtout pour assurer, par le choix des investitures, la phase suivante des élections à l'Assemblée, mais qu'il n'est nullement besoin d'incarner un besoin de renouveau en s'appuyant sur des structures préexistantes dont on a patiemment conquis au préalable les forces et tissé à son profit les réseaux.

Ce cursus politique «classique» où la conquête du parti précède les candidatures, où les primaires déterminent le choix des candidats est, en 2017 , périmé. Le «régime des partis» est donc bel et bien dépassé. 
Pour autant, ces variations fortes quant au poids des appareils partisans dans le processus électoral, dans la reprise des attentes sociétales, dans la défense d'idées, comme le déclin de l'activité militante, semblent sans impact sur la place institutionnelle ou le statut des partis.

Celui-ci est construit sur le fondement de la liberté individuelle : liberté d'opinion, et donc liberté d'en changer, d'expression collective, de réunion pacifique ${ }^{1}$, ou encore de communication $^{2}$, liberté d'association ${ }^{3}$ (et donc de ne pas adhérer ${ }^{4}$ ), liberté de manifestation ${ }^{5}$, respect de la vie privée lorsque sont en cause la collecte et l'utilisation de données en lien avec une activité publique ${ }^{6}$, liberté pour les partis de choisir leurs responsables et d'investir discrétionnairement leurs candidats ${ }^{7}$, ou de ne pas investir une personne ${ }^{8}$ et, plus généralement, pour les partis comme pour les candidats, d'être indépendants de la puissance publique'.

L'attractivité des partis dans une sphère de droit public est donc limitée. Elle découle de leur objet et non de leur fonctionnement concret, qui ne saurait relever du contrôle du juge administratif, même en matière de contentieux électoral:

[...] le juge administratif n'est pas compétent pour vérifier la régularité de l'investiture des candidats au regard des statuts et des règles de fonctionnement des partis politiques, il lui appartient, en revanche, d'apprécier les faits révélant des manœuvres ou des irrégularités susceptibles d'avoir altéré la sincérité du scrutin ${ }^{10}$.

Autrement dit, c'est exclusivement en raison de leur participation à la vie publique, au pluralisme des courants d'opinion, et de leur rôle électoral que les partis politiques peuvent se voir assujettis à des règles spécifiques. Ainsi la Cour européenne des droits de l'homme pose-t-elle clairement une limite à l'application des droits individuels en matière d'activité politique ou syndicale:

La seule forme de nécessité capable de justifier une ingérence dans l'un de ces droits est donc celle qui peut se réclamer de la «société démocratique». La démocratie apparaît ainsi comme l'unique modèle politique envisagé par la Convention et, partant, le seul qui soit compatible avec elle ${ }^{11}$.

Il en résulte que:

[...] l'État a également le droit de prendre des mesures préventives pour protéger la démocratie face à des entités autres que des partis lorsqu'un préjudice menaçant de manière suffisamment imminente les droits d'autrui risque de saper les valeurs fondamentales sur lesquelles se fonde une société démocratique. L'une de ces valeurs est la coexistence, au sein de la société, des membres qui la composent hors de toute ségrégation raciale $[\ldots]^{12}$.

Le modèle démocratique implique le pluralisme, la liberté de création, d'adhésion et d'action des partis, dans le seul respect des valeurs qui l'établissent. Il en résulte que la liberté est la règle, l'interdiction est l'exception. La liberté ne se définit pas, seules les prohibitions doivent être strictement prévues par la loi.

\section{L'absence de définition constitutionnelle, garantie du libre exercice de l'action des partis politiques}

En 1988, où pour la première fois le législateur français établissait des règles pour régir le financement des partis politiques, le gouvernement comme l'opposition parlementaire, sensible à cette forte détermination de liberté individuelle et à cette faible limite de respect de valeurs démocratiques, n'ont pas souhaité saisir le juge constitutionnel de la loi ordinaire du 11 mars 1988. Il en résulte que les partis politiques relèvent du droit privé, dans le cadre de garanties des libertés individuelles que celui-ci définit. Il en résulte aussi que le cadre financier défini par ces lois est proposé mais ne saurait être imposé. Il en résulte enfin qu'un parti n'est pas juridiquement défini.

Depuis 1988, si la «condition économique des partis politiques» (pour emprunter cette expression à Yves Poirmeur ${ }^{13}$ ) a sensiblement évolué, si leur libre participation au titre des recettes d'une campagne électorale est explicitement reconnue par l'article L. 52-8 du Code électoral, les principes sont restés les mêmes : le parti n'a pas besoin de se constituer pour agir. Le fonctionnement des partis, les règles d'adhésion ou d'investiture, leur discipline interne, les liens qu'ils peuvent entretenir avec un groupe parlementaire, un syndicat, une entreprise, un organe de presse ou d'édition, etc., demeurent placés sous la bannière de la liberté d'action qui leur est reconnue par l'article 4 de la Constitution, et plus généralement sous celle de la liberté d'opinion et d'expression reconnue à leurs adhérents.

La réglementation du financement est assimilée dans la jurisprudence du Conseil d'État à la possibilité d'intervenir en tant qu'organisation dans les campagnes électorales. Le Conseil d'État, après avoir tenté de dégager un critère tenant à l'objet et aux conditions de l'activité du groupe en

1. Cour EDH, 9 décembre 2013, Vona c. Hongrie, nº 35943/10.

2. En dernier lieu, pour les campagnes électorales, voir CC, déc. nº 2017-752 DC du 8 septembre 2017.

3. Cour EDH, 12 janvier 2016, DTP c. Turquie, $\mathrm{n}^{\circ} 3840 / 10$.

4. Cour EDH, 29 avril 1999, Chassagnou et autres $c$. France, no $25088 / 94$

5. CC, déc. $\mathrm{n}^{\circ}$ 95-395 DC du 18 janvier 1995.

6. CC, déc. $\mathrm{n}^{\circ}$ 2017-637 QPC du 16 juin 2017.

7. $\mathrm{CE}, 11$ mai 2005 , Élections municipales de Clichy, $\mathrm{n}^{\circ} 386018$.

8. CC, déc. $\mathrm{n}^{\circ}$ 2012-4598 AN du 7 décembre 2012.

9. CC, déc. $\mathrm{n}^{\circ}$ 88-242 DC du 10 mars 1988

10. CE, 11 mai 2005, Élections municipales de Clichy, cons. 10.

11. Cour EDH, 30 janvier 1998, Parti communiste unifié de Turquie c. Turquie, $\mathrm{n}^{\circ} 19392 / 92, \$ 45$.

12. Cour EDH, 9 décembre 2013, Vona $c$. Hongrie, $\$ 57$.

13. Y. Poirmeur, Les partis politiques: du XIXe au XXIe siècle en France, Issy-les-Moulineaux, LGDJ, 2014, p. 12. 
cause $^{14}$, s'en est tenu à un critère d'apparence tautologique, en posant cette identité avec l'arrêt Élections municipales de Fos-sur-Mer ${ }^{15}$. Est parti politique au sens du financement des campagnes électorales ce qu'est un parti politique au sens de la réglementation financière générale, donc concrètement une entité relevant de la compétence de la Commission nationale des comptes de campagne et des financements politiques (CNCCFP).

Cette évolution jurisprudentielle traduit bien une renonciation à une définition juridique du parti en fonction de ses finalités. Cette renonciation paraît triplement fondée.

D'abord parce que tenter de définir un parti comme une organisation destinée à exercer le pouvoir, donc supposée proposer un projet global de société, donc animée par une vision globale de la société, si cela correspond à une définition intellectuellement acceptable, est loin d'être suffisant en termes juridiques. Tel groupement de défense d'intérêts, avec un objet limité, peut donc évoluer vers la sphère partisane: on en connaît de multiples cas, illustrés par exemple par la candidature de Jean Saint-Josse à l'élection présidentielle, qui obtient 4,23\% des suffrages le 21 avril 2002 avec des thèmes de campagne très sectorisés.

Ensuite parce que la liberté ne se cantonne autrement que par des restrictions légales. La libre constitution et activité des partis impose donc que ne soient jugées que les illégalités qu'ils commettraient ou les irrégularités de fonctionnement internes aux règles qu'ils se sont eux même fixées, sur la base de la liberté d'adhésion de ses membres et de la libre acceptation de ces adhésions par les structures internes de ces partis.

Enfin, parce que la seule limite à l'activité des partis tient au respect des valeurs d'une société démocratique, «unique modèle» des sociétés contemporaines comme le juge la Cour européenne des droits de l'homme. Cette restriction est légitime selon l'article 4 de la Constitution française ou l'article 21 de la loi fondamentale allemande. Cette seule restriction a deux types de conséquences, l'une tenant à la possible ingérence de la sphère publique, ou, plus exactement dit en termes juridiques, de l'ordre public, l'autre à l'impossibilité de reconnaître une quelconque exclusivité aux actions partisanes.

Au titre de l'ingérence nécessaire, on peut citer l'article L. 212-2 du Code de la sécurité intérieure, dont l'origine remonte à la loi du 10 janvier 1936, texte qui ne vise d'ailleurs pas les partis politiques, mais les associations ou groupements de fait dont l'activité est contraire à cet «unique modèle», en particulier à la «légalité républicaine». Ce texte permet ainsi d'interdire des groupes de supporters extrémistes, un groupe qui diffuse « un islamisme radical, marqué par une forte hostilité à l'égard des chrétiens, des juifs et des chiites, prônant un rejet des valeurs et de certaines lois de la République», affichant «leur soutien au djihad armé», éléments qui "caractérisent l'existence de discours et de faits provoquant à la discrimination, à la haine ou à la violence» ou les justifiant, et «en dépit d'attestations de fidèles de la mosquée réfutant l'existence de prêches à caractère radical ${ }^{16}$.

La contrepartie est que les partis ne peuvent disposer d'aucun monopole d'action: les candidatures, y compris pour l'élection présidentielle, ne nécessitent pas une présentation par un parti, un groupe politique dans l'une des chambres du Parlement n'est pas nécessairement le correspondant d'un parti, etc.

Il en résulte que, comme le reconnaît le gouvernement en réponse à une question écrite portant sur un parti dont le caractère religieux est revendiqué, il n'y a pas de définition juridique du parti politique mais seulement un cadre légal dans lequel il peut choisir d'entrer:

L'article 4 de la Constitution de 1958 dispose que «Les partis et groupements politiques concourent à l'expression du suffrage. Ils se forment et exercent leur activité librement. Ils doivent respecter les principes de la souveraineté nationale et de la démocratie [...]». Pour autant, il n'existe pas de définition juridique du parti politique. La législation relative à la transparence et le financement de la vie politique permet toutefois de préciser cette notion, sur le fondement de critères comptables et financiers. [...] Toute association peut présenter des candidats aux élections sans pour autant être un "parti politique» au titre de la loi [...]. [...] Dans ce cas, les candidats déclarent simplement représenter telle ou telle autre association. En revanche, si l'association ne se soumet pas aux règles de la loi du 11 mars 1988, elle ne peut financer la campagne de ces candidats. [...] ni la Constitution ni la loi du 9 décembre 1905 concernant la séparation des Églises et de l'État ne limitent le droit d'expression des associations politiques en matière cultuelle ou de laïcité ${ }^{17}$.

Ainsi, il n'existe pas de définition légale, ni même juridique, du parti politique, mais seulement un cadre légal proposé par la loi ou une prohibition légale d'objets ou d'activités interdits.

\section{Les partis politiques, organismes de droit privé, entretiennent des rapports de droit privé avec leurs membres}

Il en résulte que les relations internes aux partis politiques, c'est-à-dire les relations entre les partis politiques et leurs membres ne peuvent être appréhendées que par le juge judiciaire. Elles relèvent, comme l'adhésion ou le militantisme, de la sphère privée, même si elles se caractérisent

14. CE, 31 juillet 1996, Élections municipales d'Auxerre, nº 177465, Recueil Lebon, p. 322.

15. CE, Ass., 30 octobre 1996, Élections municipales de Fos-sur-Mer, Revue française de droit administratif, 1997, concl. L. Touvet, p. 59; Petites affiches, 7 mars 1997, p. 14, note J.-P. Camby. Voir aussi CE, 10 juillet 2015, no 388623 , L'actualité juridique. Droit administratif, 2016, note E. Forey, p. 440.

16. CE, 26 janvier 2018, Association Rama de Torcy, $\mathrm{n}^{\circ}$ 412312, cons. 4 et 5. Pour d'autres exemples, voir J.-P. Camby, «Les partis politiques peuvent-ils avoir un juge?», in Le droit interne des partis politiques, J. Benetti, A. Levade, D. Rousseau (dir.), Paris, Mare \& Martin, 2017, p. 129 sq. Voir aussi, CE, 26 janvier 2018, n 407220, et par exemple pour un groupe faisant l'apologie de la lutte armée et ayant installé une école coranique clandestine: CE, 26 juillet 2016, $\mathrm{n}^{\circ} 401379$.

17. Réponse à la question écrite de G. Chevrollier n 22851, Journal officiel de l'Assemblée nationale, 10 septembre 2013. 
par une action collective ou publique. C'est en dernier lieu avec l'affaire de la présidence d'honneur du Front national, que la Cour de cassation, le 25 janvier 2017, s'est prononcée sur la question, en des termes tout à fait explicites, qui écartent tout traitement contentieux spécifique tenant à la nature de l'activité «politique» de ces entités:

Attendu que le Front national fait grief à l'arrêt de déclarer la juridiction judiciaire compétente pour connaître du litige, alors, selon le moyen, que, suivant l'article 4 de la Constitution, les partis et groupements politiques concourent à l'expression du suffrage; qu'ils se forment et exercent leur activité librement et doivent respecter les principes de la souveraineté nationale et de la démocratie; qu'ainsi, investis d'une mission de service public, les litiges intéressant la mise en œuvre de leur règlement intérieur ressortissent à la compétence de la juridiction administrative; qu'en décidant du contraire, la cour d'appel a excédé ses pouvoirs et violé la loi des 16-24 août 1790; mais attendu que, si les partis et groupements politiques concourent à l'expression du suffrage et jouent un rôle essentiel au bon fonctionnement de la démocratie, le principe de liberté de formation et d'exercice qui leur est constitutionnellement garanti s'oppose à ce que les objectifs qu'ils poursuivent soient définis par l'administration et à ce que le respect de ces objectifs soit soumis à son contrôle, de sorte qu'ils ne sauraient être regardés comme investis d'une mission de service public; que l'arrêt relève que le Front national est une association de droit privé, régie par les dispositions de la loi du $1^{\text {er }}$ juillet 1901; qu'il en résulte que le litige qui l'oppose à l'un de ses membres ne peut relever que de la compétence de la juridiction judiciaire $[\ldots]^{18}$.

La seule grille de lecture juridique de l'action des partis politiques, dès lors que leur fonctionnement ne heurte pas l'ordre public, la démocratie, la légalité républicaine et la souveraineté nationale, condition qui, pour les partis politiques connus au plan national, est satisfaite, relève donc du juge judiciaire, en fonction des critères qui sont les siens. Il doit apprécier si les rapports de droit privé définis par l'adhésion, la participation à l'activité, la direction de celle-ci ou encore la discipline interne ou l'exclusion sont ou non réguliers.

Ainsi, la même affaire que celle qui vient d'être citée a donné lieu, plus récemment et pour des faits incriminés distincts, au jugement de la cour d'appel de Versailles du 9 février dernier:

Considérant que la décision d'exclusion d'un adhérent d'un parti politique n'interdit pas à celui-ci d'exercer son activité; qu'elle n'a pas de conséquence professionnelle; qu'elle est uniquement fondée sur la violation du contrat liant les membres de l'association [...] ceux qui décident l'exclusion sont également parties au contrat d'association [...]. Considérant que le bureau exécutif siégeant en formation disciplinaire n'est pas une juridiction; que les dispositions procédurales régissant la récusation devant les juridictions civiles ou pénales ne sont donc pas applicables [...] il ne ressort d'aucun principe général du droit que les membres de la formation disciplinaire d'une association politique chargée d'examiner des manquements au contrat liant ses membres sont soumis aux règles d'impartialité dégagées pour des juridictions; Considérant qu'il ne résulte d'aucune disposition légale ou réglementaire une quelconque obligation faite à une association politique de prévoir une faculté de récusation; Considérant qu'à défaut de principe général du droit ou de dispositions légales ou réglementaires permettant la récusation de membres de l'instance chargée d'examiner des manquements au "pacte associatif», cette faculté de récusation ne peut résulter que des statuts de l'association elle-même; Considérant que ni les statuts de l'association Front national ni son règlement interne ne prévoient cette faculté; Considérant que les statuts et le règlement régissant les relations entre ses membres n'ont donc pas été violés ${ }^{19}$.

Cet arrêt illustre bien la délimitation des activités «privées » des partis politiques, à laquelle se cantonne le juge. Elles ne sauraient s'étendre au-delà de l'activité du parti lui-même, en particulier avoir des conséquences professionnelles. D'ailleurs le Code du travail interdit les discriminations liées aux opinions politiques, qu'elles concernent l'embauche (art. L. 1132-1) ou le contenu du règlement intérieur (art. L. 1321-3). Ces activités définissent seulement un lien juridique entre adhérent et parti, de nature contractuelle ${ }^{20}$

L'adhésion à un parti politique entraîne donc seulement un «pacte associatif» ; le fait d'y jouer un rôle de direction, ou d'en être le candidat, repose sur le même fondement juridique. Elle n'impose au parti, ni naturellement à ses membres, quel que soit leur rôle, aucune sujétion de service public, et les conditions d'exercice de la discipline interne ne résultent, à leur tour, que de ce pacte associatif.

Les partis ont pour finalité de décider d'une action politique, qu'on pourrait, faute de mieux, qualifier d'action tournée vers la sphère publique, à la conquête d'un pouvoir, action qui peut être publique ou stratégiquement secrète, élective ou non. Cette finalité ne peut être définie juridiquement, mais seulement garantie, par le silence même de la loi.

Il en résulte que les partis politiques sont des entités de droit privé, libres de se constituer et d'agir comme elles le souhaitent, sous réserve du respect de la légalité. Dans son versant public, cette légalité fait obstacle à des partis niant la démocratie ou la souveraineté nationale.

Dans son versant privé, largement dominant dans le fonctionnement des partis, elle postule que les relations entre les adhérents du même organisme soient équitables, et respectueuses du pacte associatif sur lequel repose l'adhésion. Dès lors que les statuts qui traduisent ce pacte sont respectés, l'adhésion volontaire, ou les règles de radiation, ne sauraient être jugées en fonction de la finalité politique de la structure: une organisation politique est, au sens juridique, une organisation humaine, collective, régie à ce titre par le seul droit privé.

18. CC, arrêt $\mathrm{n}^{\circ} 102$ du 25 janvier 2017, 15-25/561. Voir P. Jan: "Les partis politiques n'exercent pas une mission de service public», La semaine juridique, édition générale, $\mathrm{n}^{\circ} 11,13$ mars $2017,270$.

19. CA Versailles, 9 février $2018, \mathrm{n}^{\circ} 16 / 085411$.

20. Voir dans le même sens TGI de Paris, 19 mai 2015, à propos de la présidence du Parti radical. 